\title{
Experiencia en el uso video-Impulso Cefálico (vHIT) en la evaluación del reflejo vestíbulo-ocular para el canal semicircular horizontal
}

\section{Assesing vestibulo-ocular reflex of the horizontal semicircular canal with video Head Impulse Testing (vHIT)}

Hayo Breinbauer K', José Luis Anabalón B², Karina Aracena C², Diego Nazal², María de los Ángeles Baeza A'.

\section{RESUMEN}

Introducción: La prueba de impulso cefálico consiste en una herramienta sencilla y rápida para evaluar la función del reflejo vestíbulo ocular. La implementación de un acelerómetro y una cámara de alta velocidad, permiten registrar alteraciones sutiles de este fenómeno, optimizando esta prueba.

Objetivo: Describir la experiencia inicial y relevancia clínica de la prueba de impulso cefálico asistida por video y acelerómetro (VHIT).

Material y método: Se realizó prueba vHIT a pacientes con indicación de estudio funcional de VIII par.

Resultados: Utilizando prueba calórica como patrón de oro se evaluaron 60 pacientes. VHIT presentó una especificidad de $93 \%$ y una sensibilidad de $74 \%$. En un paciente con colesteatoma bilateral con vértigo agudo, el examen contribuyó en la identificación del lado complicado, además de registrar fenómenos de compensación vestibular.

Discusión y conclusión: vHIT demostró ser altamente específico. Los supuestos falsos positivos presentaban sintomatología vestibular, por lo que consideramos que podrían representar casos donde vHIT es más sensible que la prueba calórica. vHIT presenta además una buena sensibilidad. Los casos de falsos negativos podrían explicarse en relación a la diferencia de velocidad angular usada en la estimulación entre vHIT y la prueba calórica. vHIT podría representar un importante complemento al estudio del paciente con patología vestibular.

Palabras claves: Reflejo vestíbulo ocular, pruebas vestibulares funcionales.

\section{ABSTRACT}

Introduction: The head impulse test is a simple and quick tool for assessing the vestibuloocular reflex. The implementation of an accelerometer and a high-speed camera, allows the recording of subtle, priorly unaccessible alterations, thus optimazing the test.

Aim: To describe an initial experience and clinical relevance of a video and accelerometer assisted head impulse test (VHIT).

Material and method: We performed vHITon patients with indication of vestibular testing due to vertigo or balance related symptoms.

Results: Regarding caloric testing as gold standard, 60 patients were evaluated. vHIT had a specificity of $93 \%$ and a sensitivity of $74 \%$. In a patient with bilateral cholesteatoma with acute vertigo, vHIT examination contributed in identifying the complicated side, in addition to recording previously undetectable vestibular compensation phenomena.

\footnotetext{
Médico. Departamento Otorrinolaringología, Pontificia Universidad Católica de Chile.

${ }^{2}$ Tecnólogo Médico. Departamento Otorrinolaringología, Pontificia Universidad Católica de Chile.
} 
Discussion and conclusion: vHIT proved to be highly specific. Every supposed false positive cases had vestibular symptoms, so we consider these cases to represent a scenario where vHIT is actually more sensitive than caloric testing. vHIT showed also good sensitivity. False negative cases could be explained in relation to the test's angular velocity stimuli difference existing between VHIT and caloric test. VHIT could represent an important contribution to patients with vestibular disorders.

Key words: Vestibulo-ocular reflex, Vestibular functional tests.

\section{INTRODUCCIÓN}

Las patologías del equilibrio relacionadas con la pérdida de la función de los canales semicirculares para detectar aceleración angular tienden a ser frecuentes, intensamente sintomáticas e inhabilitantes, tanto por la ilusión de movimiento angular (fenómeno al que llamamos vértigo) como por la inestabilidad y desequilibrio que su hipofunción -habitualmente asimétrica- puede generar ${ }^{1}$.

La prueba de impulso cefálico (HIT por sus siglas en inglés, Head Impulse Test) representa una alternativa sencilla, breve, de simple interpretación y sin inducción de síntomas molestos en el paciente para la evaluación de dicha función vestibular y las patologías que la afectan ${ }^{2,3}$.

Al igual que la prueba calórica y gran parte de los estudios vestibulares, esta prueba se basa en los aspectos fisiológicos que determinan el reflejo vestíbulo-ocular (RVO). Este se encuentra dentro de los reflejos más rápidos del organismo, con una latencia de apenas 7 a 10 milisegundos, y que, para entenderlo de forma simple, se expresa en la capacidad del organismo para mantener la mirada fija en un objeto a pesar de movimientos rápidos e inesperados de la cabeza ${ }^{4,5}$.

En un paciente sano, los canales semicirculares (CSC) detectan con gran exactitud cambios en la posición de la cabeza en cualquier eje y en cualquier dirección del espacio, enviando a los músculos extraoculares las señales necesarias para generar un movimiento compensatorio del globo ocular, en sentido velocidad, perfectamente opuesto y suplementario, de modo que se genera la ilusión de que los ojos permanecen inmóviles con la vista fija en el objeto a pesar de cualquier movimiento cefálic $0^{5-7}$ (Figura 1).

Esta función es el resultado de la integración central de la información proveniente de los seis CSC, los que se organizan anatómica y funcionalmente en tres parejas. Cada pareja está conformada por un canal en cada oído, los que son "coplanares" (su disposición anatómica les hace compartir un mismo plano en el espacio) y "excitatoriamente especulares" entre sí (dentro de sus planos respectivos, un giro en un sentido genera una respuesta excitatoria en un canal e inhibitoria en su pareja, y viceversa) $)^{8,9}$. Así los canales horizontales comparten el plano horizontal, el canal anterior derecho y el posterior izquierdo comparten el plano RALP, y los canales restantes el plano LARP. Los planos RALP y LARP son conocidos también como los planos verticales. Estos tres planos son perpendiculares entre sí, logrando una cobertura ortogonal de las tres dimensiones del espacio ${ }^{4}$. La disposición del quinocilio en la ámpula de cada canal determina

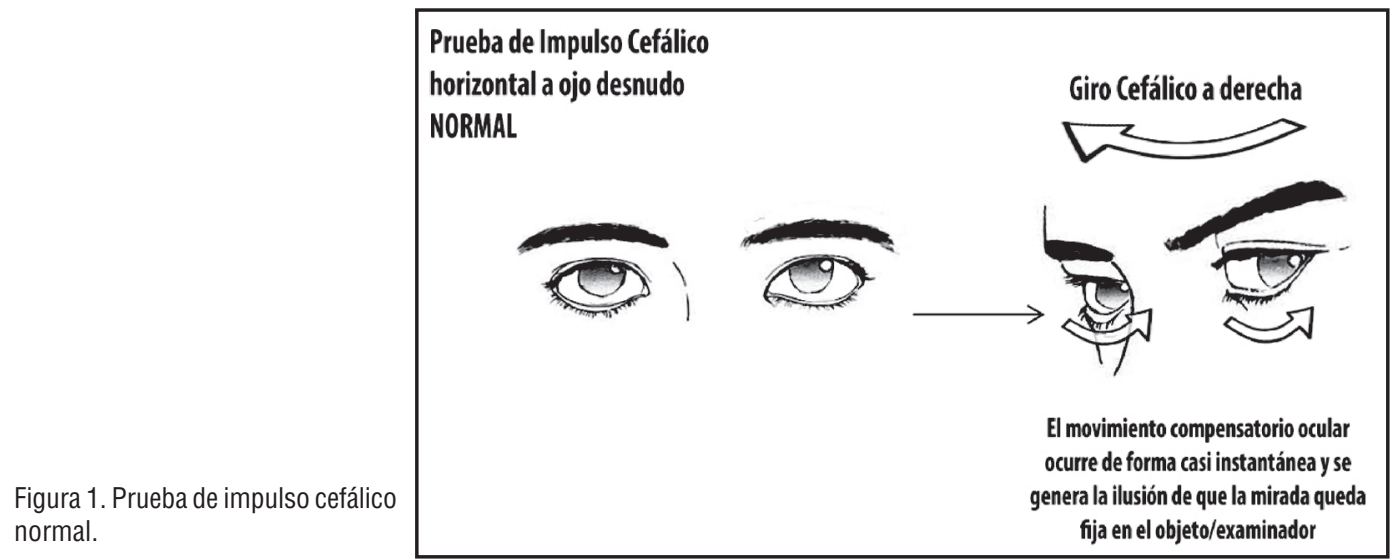


si un giro en un determinado sentido será excitatorio 0 inhibitorio. En los canales horizontales una corriente endolinfática ampulípeta (acercándose a la ámpula) generará un aumento en la tasa de descarga basal (fenómeno excitatorio). Una corriente ampulífuga generará, en contraste, una respuesta inhibitoria. Así un movimiento hacia la derecha estimulará el canal derecho e inhibirá el izquierdo. En los canales verticales ocurre lo contrario: una corriente ampulífuga es excitatoria y viceversa (giros hacia abajo-adelante estimularán los canales anteriores e inhibirán los posteriores. Lo contrario ocurre con movimientos hacia arriba-atrás) ${ }^{10}$.

A pesar de que frente a un giro cefálico se estimulan siempre ambos oídos, en los casos de gran velocidad angular existe una asimetría en la integración central de la información, lo que permite (mediante la realización de un HIT) discriminar la función de cada canal por separado, lo que diferencia este examen por ejemplo de la silla rotatoria donde no es posible conocer con certeza el lado afectado $2,8,9,11,12$.

Este fenómeno se conoce como la segunda ley de Ewald ${ }^{11}$ y se relaciona con la saturación de la información inhibitoria en los CSC. En reposo el tono basal de los CSC ronda las 70 ó 90 descargas (potenciales de acción) por segundo. Dependiendo de la aceleración angular a la que se vean expuestos, esta tasa puede subir a más de 350 descargas/ segundo en el caso de que la endolinfa se mueva en sentido estimulatorio, lo que corresponde a una diferencia de más de 260 descargas/segundo entre tono basal y máximo estimulatorio. Sin embargo frente a un movimiento de endolinfa inhibitorio la tasa no puede disminuir por debajo de las 0 descargas por segundo, logrando un máximo de 90 descargas/segundo de diferencia entre basal y mínimo. Por mucho que aumente la aceleración angular, el lado inhibido siempre interpretará esta información como la equivalente a disminuir un máximo de 70 a 90 descargas/segundo su tono basal, saturándose la capacidad de respuesta en este sentido del canal y perdiéndose toda la información restante ${ }^{9}$.

Esta asimetría en los rangos dinámicos excitatorios e inhibitorios de respuesta, produce que frente a aceleraciones angulares que generan cambios dinámicos de más de 70-90 descargas/segundo sea exclusivamente la información proveniente del canal estimulado (que tiene un mayor rango dinámico) la que predomine como aferencia del sistema en su integración central'12.

Esta característica explica la principal diferencia entre la prueba de impulso cefálico y otras pruebas donde se somete al paciente a aceleración angular, como por ejemplo la silla rotatoria. En esta última el movimiento angular oscila entre 0,01 a $1,3 \mathrm{~Hz}$ (los movimientos angulares pueden ser analizados en términos de velocidad angular $(\%)$, aceleración angular $\left(\% / s^{2}\right) 0$ frecuencia angular $(\mathrm{Hz})$. Este último término -aunque siendo el más confuso- es uno de los más usados en la literatura dentro del estudio de movimientos cefálicos y puede entenderse como la "cantidad de iteraciones o giros por segundo" que podrían realizarse con una aceleración angular máxima determinada) estímulo insuficiente para superar el nivel de saturación inhibitoria de los CSC, lo que hace imposible determinar con total certeza cuál es el oído enfermo ${ }^{13,14}$. La prueba de impulso cefálico por otro lado, consta de movimientos cortos de altísima velocidad $(2 \mathrm{a} 7 \mathrm{~Hz}$ ) que supera con creces este umbral, permitiendo una evaluación de cada uno de los seis canales por separad $0^{8,15}$.

Cuando los movimientos cefálicos son pasivos (generados por un examinador y no por el paciente), e inesperados (no se ciñen a una pauta que el paciente pueda anticipar) se minimizan otras aferencias que modulan el reflejo vestíbulo-ocular (reflejos cérvicooculares, función oculomotora voluntaria o facilitación desde eferencias corticales o cerebelosas), permitiendo una evaluación exclusiva de la función de los CSC ${ }^{16,17}$.

En pacientes con patología la respuesta del RVO frente a un movimiento cefálico se retrasa, siendo necesaria una sacada correctiva (semejante a la fase rápida del nistagmo) para reposicionar el ojo hacia el objetivo que se está mirando. Durante el examen este retraso se percibirá como un "error" en la posición ideal del ojo con respecto al blanco que se sigue, donde se observará que el ojo "sigue a la cabeza" desviando la mirada hacia un punto lejano ${ }^{4}$. Un instante después (100 a 300 milisegundos), el ojo se moverá para redirigir la mirada hacia el objeto ${ }^{17}$ (sacada correctiva) (Figura 2).

Esta sacada es visible a ojo desnudo en muchos casos, pero puede pasar inadvertida cuando ocurre durante el movimiento de la cabeza (sacadas "cubiertas" o covert-saccades en la literatura) y no después de éste ${ }^{18-20}$ (sacadas descubiertas 0 overt-saccades). Es en esta situación donde resulta necesario incorporar no sólo un sistema de medición objetivo y confiable para el movimiento del globo ocular, sino también un acelerómetro para registrar el giro de la cabeza.

Previamente, y sólo aplicable en una situación de laboratorio, la estrategia "patrón de oro" para registrar el desplazamiento ocular se basaba en la instalación de bobinas electromagnéticas adosadas como un lente 


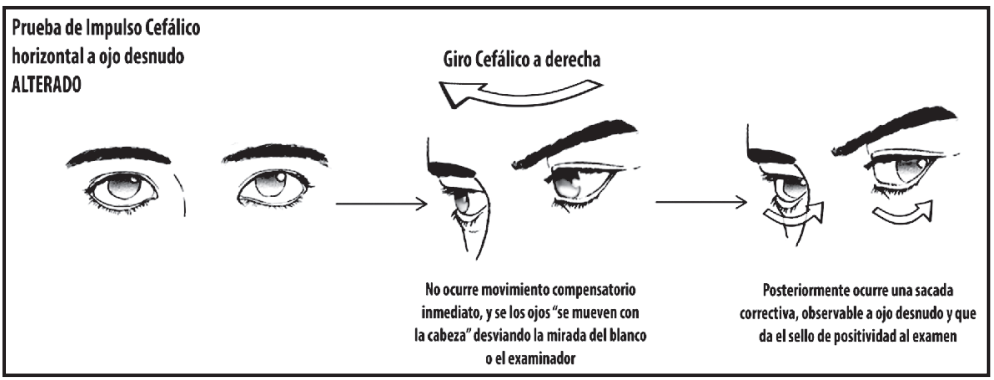

Figura 2. Prueba de impulso cefálico normal.

de contacto en la esclera del globo ocular del paciente $^{17,21,22}$. Esta metodología tiene varias limitaciones, que incluyen tanto una no despreciable incomodidad para el paciente, como una gran dificultad y tiempo necesarios para la calibración de la detección de la bobina escleral dentro de un campo electromagnético.

Más recientemente ha surgido la posibilidad de detectar los movimientos oculares con una cámara ultraliviana de alta velocidad adosada a gafas, las que además tienen un acelerómetro incorporado. Esta tecnología, conocida como video Head Impulse Test (vHIT) ha demostrado una sensibilidad y especificidad de $100 \%$ al compararla con la técnica de bobinas esclerales ${ }^{19,20,23}$, convirtiéndola en una forma simple de evaluar el RVO con gran detalle.

Utilizando gafas semejantes a las mostradas en la Figura 3, se le solicita al paciente mantener la vista fija en un punto colocado aproximadamente a un metro de distancia. El examinador se coloca detrás del paciente y con sus manos sujetando ambos lados de la cabeza de éste, genera movimientos -denominados "impulsos"- rápidos, aleatorios (tanto en dirección como intervalo entre impulsos), inesperados, sin rebote (movimiento "seco" hacia un lado, sin regresar a línea media) y de gran velocidad angular en el plano de los CSC que se pretendan evaluar. Por ejemplo para los canales horizontales (los más sencillos de evaluar) se inclina la cabeza hacia adelante en $30^{\circ} \mathrm{y}$, siempre comenzando con la mirada en línea media con respecto al punto de referencia frente al paciente, se gira la cabeza entre $10^{\circ}$ y $15^{\circ}$ hacia los lados (si se gira la cabeza hacia la izquierda se está estimulando el CSC izquierdo). Para los canales verticales la posición inicial es con la cabeza desviada $35^{\circ}$ a $45^{\circ}$ hacia un lado (con el paciente fijando la vista en el punto de reojo) de forma de alinear el plano RALP o LARP con dicho punto, para luego realizar un impulso hacia arriba-atrás (para estimular el canal posterior) o hacia adelante-abajo (para estimular el canal anterior). Habitualmente son suficientes alrededor de 20 impulsos hacia cada lado para obtener un registro confiable ${ }^{3,4,19,20,23}$.

En cada impulso, el vHIT registra tanto el movimiento de la cabeza, como el del ojo, entregando dos curvas de velocidad angular en el tiempo (Figura 4). El movimiento del ojo siempre ocurre en dirección opuesta al de la cabeza, sin embargo algunos sistemas de VHIT (como el utilizado en esta experiencia) invierten una de las curvas, con el fin de poder comparar la similitud de ambas con mayor facilidad. Cada curva puede además sintetizarse en un valor resumen de velocidad angular, como por ejemplo el área bajo la curva, o la velocidad puntual a un determinado tiempo desde el inicio del impulso, etc ${ }^{19,20,23}$.

La relación entre ambas velocidades da cuenta de la "ganancia del RVO". Valores de ganancia cercanos a uno (la respuesta del ojo es prácticamente idéntica al movimiento de la cabeza, donde observaremos curvas de velocidad muy semejantes) hablan de un reflejo conservado, mientras valores cercanos

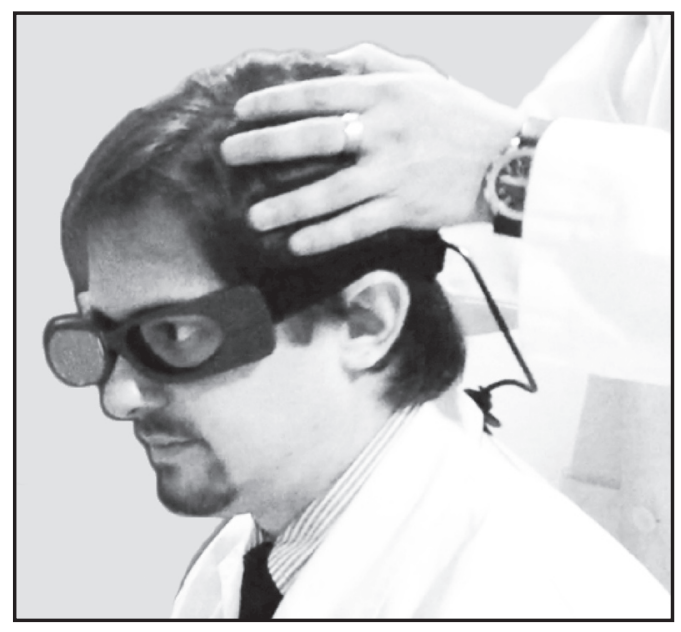

Figura 3. Video HIT. 
a cero traducen un reflejo patológico. En este caso es esperable observar una segunda elevación, muy rápida y breve de la velocidad ocular y que representa una sacada correctiva. Siempre que ocurre una de estas sacadas es porque hubo un "error" entre la posición real del globo ocular y la posición esperada del globo ocular para mantener la mirada fija en el punto a observar durante el examen ${ }^{24}$. Es posible esperar sacadas pequeñas de menos de 100 $\%$ s en menos del $20 \%$ de los impulsos en pacientes normales ${ }^{20}$. Sacadas de mayor velocidad 0 más frecuentes hablarían de un error mayor o más frecuente del reflejo vestíbulo-ocular, traduciendo un grado de patología y siendo uno de los criterios de hallazgos de alteración en un vHIT (Figura 4).

Como ya mencionamos estas sacadas pueden ocurrir después de completarse el movimiento de la cabeza, o durante éste. Las primeras se denominan sacadas overt y pudiesen ser más fácilmente detectadas a ojo desnudo (Figura 5). Las segundas ocurren también dentro del movimiento de la cabeza y son imperceptibles a ojo desnudo. De momento no hay claridad sobre la diferencia fisio- lógica de estos dos tipos de sacadas. Se plantea que pudiesen originarse en porciones distintas de la sustancia reticular encargada de generar movimientos correctivos y que su diferencia y latencia de aparición podría estar relacionada con procesos de compensación vestibular ${ }^{25}$. Sin embargo ambas se consideran igualmente patológicas y pueden aparecer en cualquier momento de la historia de una hipofunción vestibular.

En síntesis, el vHIT consiste en la aplicación de una prueba simple, rápida, objetiva y que no induce síntomas molestos en el paciente para evaluar la función de cualquiera de los seis CSC por separado.

\section{OBJETIVO}

En el presente trabajo se presenta la experiencia inicial del uso de vHIT en pacientes con sintomatología vestibular, enfocándonos en describir los elementos más significativos dentro de nuestro aprendizaje en esta novedosa e interesante herramienta (inicialmente sólo para el canal horizontal).

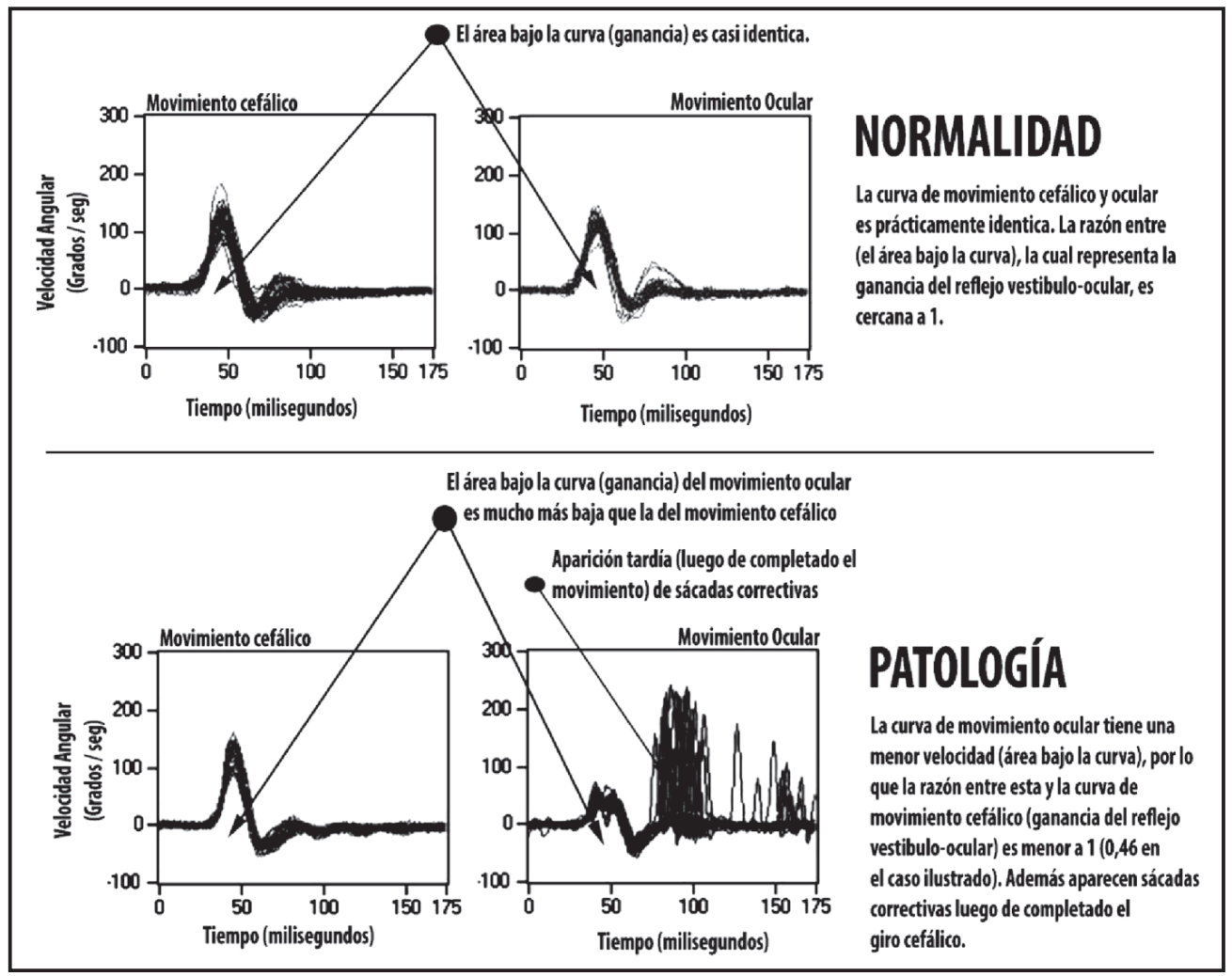

Figura 4. Interpretación de registro de vHIT. 


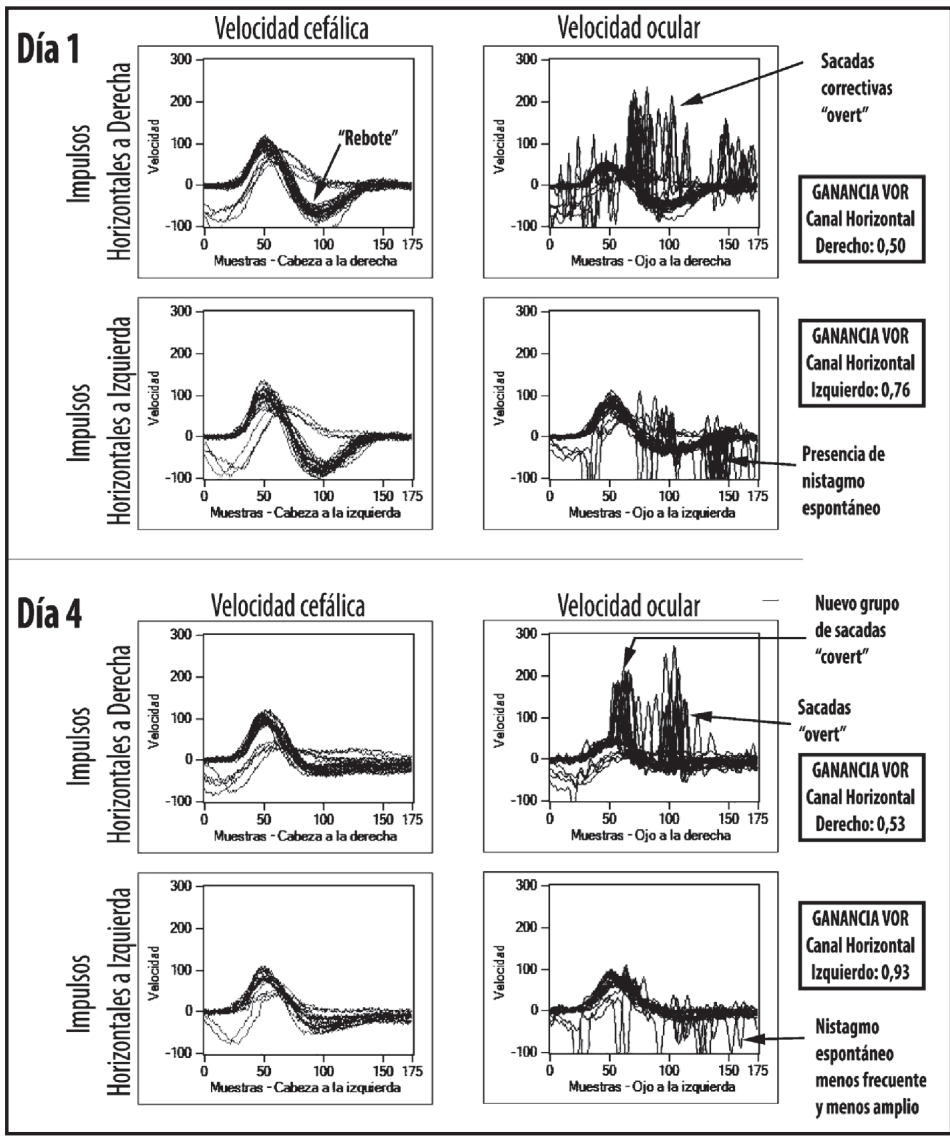

Figura 5. Caso de paciente con colesteatoma complicado en primer y cuarto día de evolución.

Además, se realizó un análisis comparativo con el patrón de oro habitual para la función vestibular en nuestro medio: la prueba calórica.

\section{MATERIAL Y MÉTODO}

Se realizó un estudio prospectivo de evaluación de test diagnóstico entre agosto y noviembre de 2012, en la Red de Salud UC.

Se escogió una muestra por criterio de factibilidad (no - probabilístico), que consistió en pacientes con indicación de estudio funcional de VIII par. Se consideraron como criterios de exclusión la presencia de estrabismo o pérdida severa de agudeza visual en el ojo derecho (limitación de método de registro del equipo utilizado, el cual sólo posee una cámara para el lado derecho).
Previo consentimiento informado, se realizó en todos los pacientes prueba de impulso cefálico asistida por video para el canal horizontal, utilizando equipo ICS Impulse (GN Otometrics, 2012).

Para dicha aplicación, se colocaron las gafas del equipo ajustadas firmemente en el paciente (ver Figura 3), el cual se mantuvo sentado aproximadamente a un metro de distancia de una pared, en la cual se había colocado una marca ligeramente por debajo de su horizonte visual (no son necesarias distancias estrictas).

Durante la realización del examen se prestó atención en mantener la cabeza del paciente inclinada en aproximadamente $30^{\circ}$, pretendiendo alinear los canales horizontales en un plano paralelo al del impulso.

Luego de una calibración automática inicial del equipo (donde se optimiza el registro del mo- 
vimiento ocular), y siguiendo recomendaciones de los fabricantes y de la literatura, se procedió a realizar al menos 20 impulsos cefálicos en cada dirección, procurando que cada impulso fuese rápido (entre $150 \%$ y $250 \%$ s de velocidad angular máxima - esta información es retroalimentada al examinador en tiempo real por el equipo para ajustar la realización de la maniobra), corto (no superando los $15^{\circ}$ a $20^{\circ}$ de desviación desde la línea media hacia cada lado), inesperados y aleatorios en cuanto a su dirección (se puso cuidado en no seguir un patrón regular o predecible en cuanto a dirección e intervalo entre los impulsos).

Se registraron y analizaron tanto forma de las curvas representando movimiento ocular, como Ios valores cuantitativos de ganancia del RVO, calculado según área bajo la curva del movimiento ocular principal (un algoritmo del programa de análisis descarta el área bajo la curva de sacadas correctivas o de nistagmo espontáneo).

Sin pretender establecer valores definitivos de sensibilidad y especificad para este test (considerando el presente estudio sólo como una experiencia inicial, por lo que no se determinó un tamaño muestral necesario para estimar hallazgos determinantes), se calcularon dichos valores considerando los resultados de la prueba calórica como patrón de oro.

El análisis estadístico se realizó con software estadístico SPSS 20.0.

\section{RESULTADOS}

Se evaluaron dentro del estudio a 60 pacientes entre 11 y 68 años de edad, donde el $54 \%$ fueron mujeres y el $62 \%$ presentó una prueba calórica alterada. El tiempo promedio de realización de vHIT fue de 4 minutos con un máximo de 7 minutos. En ningún caso el paciente manifestó incomodidad, mareo 0 vértigo.

Considerando como patológicas la presencia de sacadas correctivas o una ganancia del RVO menor a 0,6 (valores sugeridos) ${ }^{23}$, la especificidad de vHIT con respecto a hallazgos de anormalidad en la prueba calórica fue de 93\%. Los cuatro casos de falsos positivos presentaba al momento del examen vértigo e inestabilidad dentro de su sintomatología.

En términos de sensibilidad vHIT alcanzó $73 \%$, donde $60 \%$ de los falsos negativos presentaron va-
Iores de ganancia del VOR inesperadamente altos (sobre una relación de ganancia de 1,2).

Destaca dentro de nuestra experiencia el caso de un paciente de 58 años hospitalizado por vértigo agudo de 24 hrs de evolución en el contexto de colesteatoma bilateral, en cuyo caso vHIT mostró signos claros de hipofunción vestibular derecha, lo que clínicamente correspondió dentro de su evolución y manejo con el lado efectivamente complicado. A pesar de presentar vértigo e inestabilidad intensa durante toda su hospitalización, el paciente no manifestó ningún incremento de sus síntomas durante la realización del examen, el cual fue muy bien tolerado y repetido diariamente en su cama de hospitalización como ensayo de monitorización. La Figura 5 muestra los resultados de su examinación al primer y cuarto día de evolución.

En el día 1 observamos una disminución importante de la ganancia del RVO al lado derecho $(0,50)$ y la aparición clara de sacadas overt en las curvas para el canal horizontal derecho, ambos indicadores claros de patología. En el día 4 además de mantenerse una ganancia patológica, se aprecia la aparición de sacadas más precoces, incluso dentro del movimiento cefálico (covert).

En las figuras de velocidad cefálica se aprecian además algunos errores más frecuentes dentro del aprendizaje en el uso de esta técnica. Por un lado la velocidad máxima alcanzada está por debajo de los $150^{\circ}$ a $250^{\circ} / \mathrm{s}$ óptimos. Además se observa una pequeña curva de "rebote" que indica un movimiento de "ida y regreso" a línea media, en vez de un impulso "seco" hacia un lado sin retorno, que sería el ideal (y que sí se logra en la evaluación del día 4).

Adicionalmente se observan claramente en las curvas para impulsos a la izquierda algunas sacadas hacia "abajo" en el registro (más abundantes y de mayor amplitud en el día 1, y menos frecuentes y pequeñas en el día 4). Estas espigas representan la presencia de nistagmo espontáneo del paciente, y aparecen hacia abajo ya que éstas ocurren en dirección opuesta al movimiento compensatorio del ojo, al realizar el impulso hacia este lado. El nistagmo espontáneo también está presente en los impulsos a derecha, pero al ocurrir éstos en la misma dirección que el movimiento compensatorio y las sacadas correctivas tras el impulso imperfecto, son mucho más difíciles de leer dentro del registro. 


\section{DISCUSIÓN}

Este último caso comentado sintetiza las principales ventajas que este nuevo examen podría tener, y se refieren a la posibilidad de evaluar, sin la generación de síntomas molestos, la función vestibular de cada CSC por separado, incluso en una situación aguda, de forma rápida, portátil y fácilmente repetible. Más aún queda en evidencia como una alteración severa de oído medio como un colesteatoma 0 una perforación bilateral no representan un impedimento.

Hemos escogido este caso ya que además de reflejar algunos de los errores iniciales más habituales en la técnica de generación de los impulsos (falta de velocidad y rebote en el impulso), evidencia dos fenómenos especiales propios de las etapas agudas de una hipofunción vestibular y de los mecanismos de compensación tempranos.

En el día 1, no solo se aprecia una disminución de la ganancia en niveles patológicos para el lado afectado (ganancia derecha de 0,5), sino que se observa además una disminución parcial de ganancia en el lado sano $(0,76)$, sin la aparición de sacadas correctivas. Este fenómeno está descrito en las etapas iniciales de la pérdida de función del $\mathrm{CSC}^{8}$, y representa la ausencia de la contribución inhibitoria del lado enfermo al reflejo vestíbuloocular cuando se estimula el lado sano. Dada la asimetría en favor de las respuestas excitatorias, esta disminución es compensable por el lado sano (aunque el reflejo ocurre algo más lento no alcanza a generar un error que deba ser corregido por una sacada). Más aún, la tasa de descarga basal del lado sano se incrementa dentro de los primeros días, para compensar de forma más eficiente la hipofunción contralateral, lo que se observa en la recuperación a niveles normales de la ganancia del RVO en el lado izquierdo al cuarto día de evolución (ganancia de 0,93).

Por otro lado hay una clara diferencia en el comportamiento de las sacadas correctivas entre el primer y cuarto día. En un comienzo todas las sacadas se agrupan posteriores al final del movimiento cefálico, pero al cuarto día se reconocen claramente dos grupos de sacadas, uno mucho más precoz, dentro del movimiento cefálico (covert) y uno incluso más posterior al grupo del primer día. Existe discrepancia en la literatura sobre si este fenómeno corresponde a una disminución en la latencia de respuesta de las mismas sacadas correctivas, o si las sacadas covert corresponden a sacadas "nuevas" con un origen neurofuncional distinto y con mayor velocidad de respuesta que las overt ${ }^{25,26}$.

Sea cual sea el origen de las sacadas, este fenómeno junto con la variación de la respuesta del lado sano representan parámetros de la función vestibular previamente no medibles y que pudiesen aportar importantes nociones sobre los procesos de compensación vestibular ${ }^{25}$.

Más allá de aportar estos detalles de la dinámica de la función vestibular, una de las principales virtudes del VHIT es su especificidad. Si consideramos los falsos positivos en este estudio como pacientes que efectivamente presentaban sintomatología muy sugerente de hipofunción vestibular, podemos pensar que en realidad vHIT fue más sensible que la prueba calórica en estos cuatro casos, revistiendo una especificidad real de $100 \%$.

Ahora la sensibilidad encontrada es algo menor a la publicada en otras experiencias ${ }^{19,20,23,27}$, lo que podría deberse a varios factores. Por un lado debemos considerar nuestra propia curva de aprendizaje especialmente con respecto a la adquisición de una técnica apropiada para realizar impulsos suficientemente veloces, de corta amplitud y poco predecibles para el paciente.

Otro error que realizamos frecuentemente, fue no enfatizar un ajuste suficiente de las gafas. Esta negligencia genera un artefacto, donde si las gafas se deslizan -aunque sea un par de milímetros sobre la cara del paciente- la cámara interpretará este deslizamiento como un desplazamiento de la posición de los ojos. Esto será interpretado por el sistema como un aumento artificial de la velocidad del ojo, generando valores de ganancia del RVO erróneamente altos. Este fenómeno ocurre levemente incluso con gafas bien ajustadas, por Io que valores de ganancia RVO de hasta 1,2 son tolerados como normales.

Otro factor a considerar fue nuestro aprendizaje en la lectura de los parámetros entregados por el equipo. Inicialmente asignábamos gran importancia a los valores de ganancia, pero la sola presencia de sacadas correctivas, incluso con valores de ganancia normal, deben ser considerados también como patológi$\cos ^{20,23,24,26}$ 
Finalmente es importante considerar con respecto a la baja sensibilidad, la discusión sobre si es realmente adecuado comparar VHIT con la prueba calórica, dado que la velocidad angular de estimulación de ambas pruebas es enormemente distinta. Algunos autores plantean como analogía lo poco apropiado que sería comparar una audiometría exclusiva para tonos de $250 \mathrm{~Hz}$ con una audiometría para tonos entre 1 y $4 \mathrm{Khz}^{25,26,28-30}$. Siguiendo esta misma analogía, se plantea que los rangos de estimulación correspondientes a vHIT estarían más cercanos a los que el paciente utiliza dentro de sus necesidades fisiológicas en su vida cotidiana ${ }^{27-30}$, pudiéndose incluso despreciar pruebas calóricas alteradas en presencia de un vHIT normal. Por otro lado, otros plantean que VHIT es simplemente menos sensible que la prueba calórica ${ }^{31}$.

Siendo una tecnología de aparición reciente, aún persisten muchas interrogantes sobre la aplicación de vHIT en la práctica clínica. Algunos estudios, muy prometedores, destacan la potencialidad y simpleza de VHIT en los casos de vértigo agudo para diferenciar patología central de periférica, incluso en un contexto de sala de urgencias $^{32,33}$. Otra vertiente, ya comentada, rescata la posibilidad de VHIT de objetivar el proceso de compensación y rehabilitación vestibular, mediante el análisis detallado del momento de aparición, latencia y morfología de las sacadas correctivas ${ }^{25}$.

Los autores más entusiastas consideran el complemento de vHIT junto al test subjetivo visual vertical y los potenciales miogénicos vestibulares (VEMPs, tanto oculares como cervicales) como una nueva batería integral de estudios vestibulares, los que podrían, en escaso tiempo y sin incomodar al paciente, evaluar la función de los seis canales semicirculares y los cuatro órganos otolíticos de forma independiente, siendo capaces incluso de reemplazar a la prueba calórica como parte del abordaje inicial de estudio, dejando ésta para casos particulares $25,28,34$.

A medida que aparezca nueva evidencia se irá definiendo el futuro rol de este examen dentro del estudio y manejo de los pacientes vestibulares, pero de momento éste parece ser prometedor, al menos como un sencillo, veloz y efectivo complemento dentro de la evaluación de las patologías del equilibrio.

\section{BIBLIOGRAFÍA}

1. Yin M, Ishikawa K, Wong W, Shibata Y. A clinical epidemiologial study in 2169 patients with vertigo. Auris Nasus Larynx 2009; 36: 30-5.

2. Halmagyi G, Curthors I. A clinical sign of canal paresis. Arch Neurol 1988; 45: 737-9.

3. Breinbauer H, Anabalón J. Prueba de impulso cefálico. Rev Otorrinolaringol Cir Cabeza Cuello 2011; 71: 123-30.

4. Cremer P, Halmagy G, Aw S, et al. Semicircular canal plane head impulses detect absent function of individual semicircular canals. Brain 1998; 121: 699-716.

5. Halmagy G, Curthoys I, Cremer Pet al. The human horizontal vestibulo-ocular reflex in response to high acceleration stimulation before and after unilateral vestibular neurectomy. Exp Brain Res 1990; 81: 479-90.

6. Halmagy G, CuRthoys I. Clinical testing of otolith function. Ann N Y Acad Sci 1999; 871: 195-204.

7. WuYTs F. Principle of the head impulse (thrust) test or Halmagyi head thrust test (HHTT). B-ENT 2008; 4: 23-5.

8. Strupp M, Brandt T. Vestibular Neuritis. Semin Neurol 2009; 29: 509-19.

9. Baloh R, Honrubia V, Konrad H. Ewald's second law re-evaluated. Acta Otolaryngol 1977; 83: 475-9.

10. Lasker D, Hullar T, Minor L. Horizontal vestibuloocular reflex evoked by high-acceleration rotations in the squirrel monkey, III. Responses after laberynthectomy. J Neurophysiol 2000; 83: 2482-96.

11. EWALD R. Physiologische Untersuchungen über das Endorgan des Nervus octavus. Wiesbaden: Bergmann 1892.

12. Halmagyi G, Curthoys I, Cremer P, Henderson C, M S. Head impulse after unilateral vestibular deafferentation validate Ewald's second law. J Vestib Res 1990; 1: 187-97.

13. Arriaga M, Chen D, Cenci K. Rotational chair (ROTO) instead of electronystagmography (ENG) as the primary vestibular test. Otolaryngol Head Neck Surg 2005; 133: 329-33.

14. Palomar-Asenjo V, Boleas-Aguirre M, SánchezFerrándiz N, Perez N. Caloric and Rotatory Chair Test Resuls in Patients with Ménière's Disease. Otol Neurotol 2006; 27. 
15. Halmagy G, Curthoys I, Cremer P, et al. The human horizontal vestibulo-ocular reflex to high acceleration stimulation before and after unilateral vestibular neurectomy. Exp Brain Res 1990; 81: 479-90.

16. Black R, Halmagy G, Thurtell M, Todd M, Curthoys I. The active head-impulse test in unilateral peripheral vestibulopathy. Arch Neurol 2005; 62: 290-3.

17. Halmagy G, Black R, Thurtell M, Curthoys I. The human horizontal vestibulo-ocular reflex in response to active and passive head impulses after unilateral vestibular deafferentiation. Ann N Y Acad Sci 2003; 1004: 325-36.

18. Tuernström F, Nyström A, Magnusson M. How to Uncover the Covert Saccade During the Head Impulse Test. Otol Neurotol 2012; 33: 1583-5.

19. Barti K, Lehnen N, Kohlbecher S, y C. Head impulse testing using video-oculography. Ann N Y Acad Sci 2009; 1164: 331-3.

20. Weber K, MacDougall H, Halmagy G, y C. Impulsive testing of semicircular-canal function using video-oculography. Ann N Y Acad Sci 2009; 1164: 486-91.

21. Park H, Migliaccio A, Della-Santina C, Minor L, CAREY J. Search-coil head-thrust and caloric test in Ménière's disease. Acta Otolaryngol 2005; 125: 852-7.

22. Curthoys I, Topple A, Halmagy G. Unilateral vestibular deafferentation (UVD) causes permanent asymmetry in the gain of the yaw VOR to high acceleration head impulses in guinea pigs. Acta Otolaryngol Supp/ 1995; 520: 59-61.

23. MacDougall H, Weber K, LA M, y C. The video head impulse test: diagnostic accuracy in peripheral vestibulopathy. Neurology 2009; 73 : 1134-41.

24. Pérez N, Rama-López J. Head-Impulse and Caloric Tests in Patients with Dizziness. Otol Neurotol 2003; 24: 913-7.
25. MacDougall H, Curthoys I. Plasticity during vestibular compensation: the role of saccades. Front Neurol 2012; 3: 21.

26. Blödow A, Pannasch S, Walther L. Detection of isolated covert saccades with the video head impulse test in peripheral vestibular disorders. Aurus Nasus Larynx 2012; 11: Epub ahead of print.

27. Ulmer E, Bernard-Demanze L, Lacour M. Statistical study of normal canal deficit variation range. Measuremet using Head impulse Test video system. Eur Ann Otorhinolaryngol Head Neck Dis 2011; 128: 278-82.

28. CuRThoys I. The interpretation of clinical tests of peripheral vestibular function. Laryngoscope 2012; 122: 1342-52.

29. STRUPP M. Vertigo and dizziness: the neurologist's perspective. Ophthalmologe 2013; 110: 7-15.

30. Walther L, Blödow A. Ocular Vestibular Evoked Myogenic Potential to Air Conducted Sound Stimulation and Video Head Impulse Test in Acute Vestibular Neuritis. Otol Neurotol 2013: Epub ahead of print.

31. Mahringer A, Rambold H. Caloric test and videohead-impulse: a study of vertigo/dizziness patients in a community hospital. Eur Arch Otorhinolaryngol 2013: Epub ahead of print.

32. Newman-Toker D, Saber A, Mantokoudis G, et AL. Quantitative Video-Oculography to Help Diagnose Stroke in Acute Vertigo and Dizziness - Toward an ECG for the Eyes. Stroke 2013; 44: Epub ahead of print.

33. Newman-Toker D, Kattah J, Alvernia J, Wang D. Normal head impulse test differentiates acute cerebellar strokes from vestibular neuritis. Neurology 2008; 70: 2378-85.

34. Curthoys I. A critical review of the neurophysiological evidence underlying clinical vestibular testing using sound, vibration and galvanic stimuli. Clin Neurophysiol 2010; 121: 132-44. 